Pacific Journal of Mathematics

MONOTONE EXTENSIONS OF MAPPINGS AND THEIR

J. K. Ko H 


\title{
MONOTONE EXTENSIONS OF MAPPINGS AND THEIR APPLICATIONS
}

\section{J. K. KOHLI}

\begin{abstract}
The purpose of the present paper is two-fold. On the one hand, we improve over the known techniques of extending an arbitrary mapping between topological spaces to a monotone mapping and on the other, we develop applications of the theory of monotone extensions of mappings to certain results in the folklore of literature on monotone and compact mappings. In particular, we apply the theory to improve certain results of Whyburn.
\end{abstract}

1. Introduction and motivation. For the last three decades there has been considerable interest in trying to improve the behaviour of mappings by extending their domains. The problem besides being of intrinsic interest is also important from applications viewpoint. Several aspects of the problem have been extensively explored by various authors and applications made of the techniques developed in the procress. For example, while Whyburn ([31] [33]), Bauer [2], Cain ([3] [4] [5]) investigated the problem from the viewpoint of extending a mapping to a compact mapping, Dickman ([9] [10]), Krolevec [23], Delhan and Strecker [7] have studied the problem from the aspect of perfect and closed extensions of mappings. This was followed by the work of Franklin and Kohli [12] and Kohli ([16] [17]), who studied the problem from the point of view of extending a mapping to an open mapping. More recent is the theory of monotone extensions of mappings, initiated and developed by the author in ([20] [22]).

The work of Bauer, Cain, Dickman, Franklin and Kohli, Kohli, Whyburn and others referred to in the above paragraph may be considered to be the evolution of the ideas pertaining to certain aspects of the more general problem defined in the following paragraph.

Problem. Let $P$ denote a property of mappings which is not necessarily hereditary with respect to the restrictions of mappings. In particular, $P$ may be the property of being a closed mapping, or an open mapping, or a monotone mapping, or a compact mapping, or a quotient mapping, or a perfect mapping etc. Given a function $f: X \rightarrow Y$, not necessarily continuous, from a topological space $X$ into a topological space $Y$. Does there exist a superspace $X^{*}$ of $X$ and a function $f^{*}: X^{*} \rightarrow Y$ with the following properties?

(i) $f^{*}$ possesses property $P$.

(ii) $f^{*} \mid X=f$. 
(iii) $f^{*}$ is continuous whenever $f$ is.

(iv) $X^{*}$ possesses 'nice' properties whenever $X$ and $Y$ are 'nice'.

The beautiful and elegant theory of compact and perfect extensions of mappings was initiated by Whyburn [31]. In [30] Vainstein showed that any closed mapping between metric spaces possesses a restriction which is compact and in which the image space is the same as the image of the original domain space. In view of Vainstein's result and the usual dual relationship between open and closed (sets, mappings, etc.) Whyburn was first led to anticipate that open mappings may be related in some way to compact mappings. More precisely, he anticipated that if the domain space was suitably augmented, the mapping could be extended so as to become compact, thus the given mapping would be exhibited as the restriction of a compact one. Later, this was found to be fully correct. In a paper published in 1953 Whyburn [31] showed that any mapping between Hausdorff spaces is the restriction of a compact mapping. Since then, theory has been further developed by Whyburn [33], Bauer [2], Dickman ([8] [9]), Krolevec [23], Cain ([3] [4] [5]), Delhan and Strecker [7] and others, and is sufficiently rich from applications viewpoint (see for example, ([2] [7] [8] [33])). The process of compactifying a mapping has been studied from a more general viewpoint by Dickman [9] who showed that every mapping between topological spaces is the restriction of a closed (or perfect) mapping. Once again the duality between open and closed (sets, mappings, etc.) and Dickman's result led Franklin and Kohli to anticipate and finally to prove in [12] that any mapping between topological spaces is the restriction of an open mapping. The potential of theory of open extensions of mappings developed by Franklin and Kohli [12] and Kohli ([16] [17]) is well reflected in the papers ([12] [16] [17] [18] [19]), where applications of the powerful technique of the theory are nicely illustrated.

Fairly recent is the theory of monotone extensions of mappings ([20] [22]). It is shown in ([20] [22]) that any mapping between topological spaces can be extended to a monotone mapping such that certain properties of the domain are preserved in the new domain. As such the theory is still in the process of development and is in the embryo state from applications viewpoint.

2. Hausdorff monotone extensions. There is exhibited in [20] a method of constructing a monotone extension for an arbitrary mapping such that the new domain possesses 'fairly nice' separation properties. In this section, we develop a modification of the technique of [20] for extending an arbitrary mapping to a monotone mapping. The modified construction yields a monotone extension in which the extension space is even nicer than the extension space of $[20]$ in certain situations (see Example 2.4). 
Let $f$ be a function, not necessarily continuous, from a topological space $X$ into a topological space $Y$. We say that $f$ is monotone if for each $y \in Y$ the fibre $f^{-1}(y)$ is either connected or empty. We say that a point $y \in Y$ is a non-monotone point (non-compact point) relative to $f$ if $f^{-1}(y)$ is not connected (compact). Let $M_{f}$ (respectively $C_{f}$ ) denote the set of all non-monotone (respectively non-compact) points relative to $f$. It is clear that $M_{f}$ is empty if and only if $f$ is monotone and $M_{f} \cup C_{f}$ is empty if and only if each fibre is either a continuum (= compact connected set) or empty.

Throughout the paper, the symbols $M_{f}$ and $C_{f}$ will have the same meaning as in the above paragraph, $I$ will denote the closed unit interval with the usual topology and $\mathbf{N}$ will stand for the set of natural numbers.

\section{Construction A}

Step I. For each $y \in M_{f}$, let $f^{-1}(y)=\cup\left\{C_{\alpha}: \alpha \in \Lambda_{y}\right\}$, where each $C_{\alpha}$ is a component of $f^{-1}(y)$. Let $\Lambda$ denote the disjoint union of the sets $\Lambda_{y}, y \in M_{f}$. For each $y \in M_{f}$ and each $\alpha \in \Lambda_{y}$, choose $x_{\alpha} \in C_{\alpha} \subseteq f^{-1}(y)$ and let $I_{\alpha}$ denote a copy of the closed unit interval $I$.

For each $y \in M_{f}$, let $W_{y}$ denote the disjoint topological sum of the family of the spaces $\left\{I_{\alpha}: \alpha \in \Lambda_{y}\right\}$ and let $Z_{y}$ denote the quotient space of $W_{y}$ obtained by collapsing the set $\left\{0 \in I_{\alpha}: \alpha \in \Lambda_{y}\right\}$ to a point. Let $p_{y}: W_{y} \rightarrow Z_{y}$ denote the natural mapping. Now, instead of quotient topology on $Z_{y}$ consider the topology generated by the following metric $d$ on $Z_{y}$

$$
d(s, t)= \begin{cases}|s-t|, & \text { if } s \text { and } t \text { are in the same stalk } \\ s+t, & \text { if } s \text { and } t \text { are in the different stalks. }\end{cases}
$$

The space $Z_{y}$ with the metric $d$ is a pathwise connected space. From here-onward, the space $Z_{y}$ will always be considered to be endowed with the topology induced by the metric $d$. Moreover, since for each $\alpha \in \Lambda_{y}$, $p_{y} \mid I_{\alpha}$ is an isometry in what follows, we may not distinguish between $I_{\alpha}$ and $p_{y}\left(I_{\alpha}\right)$, however, the meaning will be made clear from the context.

Step II. Let $W=X \oplus\left(\oplus Z_{y}\right)$, where the second disjoint topological sum is taken over the set $M_{f}$ of non-monotone points. Identifying each $1 \in I_{\alpha}\left(\alpha \in \Lambda_{y}\right)$ (considered as $\left.p_{y}(1) \in p_{y}\left(I_{\alpha}\right) \subset Z_{y} \subset W\right)$ with $x_{\alpha} \in$ $C_{\alpha} \subset f^{-1}(y)$ we arrive at a quotient space $X^{*}$ of $W$. The quotient mapping $q: W \rightarrow X^{*}$ composes with the inclusion mapping $i: X \rightarrow W$ to give a closed embedding of $X$ into $X^{*}$. Hence $X$ may be considered as a closed subspace of $X^{*}$. 
Let $f_{1}$ be the mapping of $W$ into $Y$ whose restriction to $X$ is $f$ and whose restriction to each $Z_{y}$ is the constant mapping onto $\{y\}$. The unique map $f^{*}: X^{*} \rightarrow Y$ satisfying $f^{*} \circ q=f_{1}$ and explicitly defined by $f^{*}(z)=f_{1}\left(q^{-1}(z)\right)$ for each $z \in X^{*}$ is a monotone extension of $f$.

To prove monotoneity of $f^{*}$, let $y \in Y$. If $y \notin M_{f}$, then $f^{*-1}(y)=$ $q\left(f^{-1}(y)\right)$ and hence connected. If $y \in M_{f}$, then

$$
\begin{aligned}
f^{*-1}(y) & =q\left(f^{-1}(y) \cup Z_{y}\right) \\
& =q\left(\left(\bigcup\left\{C_{\alpha}: \alpha \in \Lambda_{y}\right\}\right) \cup Z_{y}\right) \\
& =q\left(\bigcup\left\{C_{\alpha} \cup Z_{y}: \alpha \in \Lambda_{y}\right\}\right) \\
& =\bigcup\left\{q\left(C_{\alpha}\right) \cup q\left(Z_{y}\right): \alpha \in \Lambda_{y}\right\} .
\end{aligned}
$$

Since each of the sets $C_{\alpha}$ and $Z_{y}$ are connected, so are their continuous images $q\left(C_{\alpha}\right)$ and $q\left(Z_{y}\right)$. Again since for each $\alpha \in \Lambda_{y}, q\left(C_{\alpha}\right) \cap q\left(Z_{y}\right) \neq$ $\varnothing$, the set $q\left(C_{\alpha}\right) \cup q\left(Z_{y}\right)$ is connected. Therefore, $f^{*-1}(y)$ being the union of a collection of connected sets having non-empty intersection is connected.

Now, since a map on a quotient space is continuous if its composition with the quotient map is continuous, $f^{*}$ is continuous whenever $f$ is continuous.

Properties of the space $X^{*}$. Since only sums and quotients are used in the construction of the space $X^{*}$, any co-reflexive property [14] which is possessed by $I$ and each $Z_{y}$ will be preserved in the passage from $X$ to $X^{*}$. In particular, if $X$ is a $k$-space, or a sequential space or a chain net space [14], or a $c$-space [27], or a locally (pathwise) connected space or a sum (pathwise) connected space [21], so is $X^{*}$.

If $X$ is (pathwise) connected, so is its continuous image $q(X)$. Again, since each $Z_{y}$ is pathwise connected and for each $y \in M_{f}, q(X) \cap$ $q\left(Z_{y}\right) \neq \varnothing$. Therefore, $X^{*}=\cup\left\{q(X) \cup q\left(Z_{y}\right): y \in M_{f}\right\}$ is (pathwise) connected.

It is routine to verify that $X^{*}$ is $T_{0}, T_{1}, T_{2}, T_{3}$ and $T_{3 \frac{1}{2}}$ whenever $X$ is. We shall prove only the last case: that of complete regularity. Let $F$ be a closed subset of $X^{*}$ and suppose that $p \notin F$. If $q^{-1}(p) \cap X=\varnothing$ (where $q: W \rightarrow X^{*}$ is the quotient mapping), then $q^{-1}(p) \in Z_{y}$ for some $y \in M_{f}$. Then there is a continuous real-valued function $\varphi$ on $Z_{y}$ which is zero at $q^{-1}(p)$ and one on $\left(q^{-1}(F) \cap Z_{y}\right) \cup\left\{p_{y}(1) \in p_{y}\left(I_{\alpha}\right): \alpha \in \Lambda_{y}\right\}$. Extend $\varphi$ continuously to all of $W$ by taking it constantly one on $X$ and on each $Z_{y^{\prime}}$, $y^{\prime} \neq y$. This extended $\varphi$ defines a continuous real-valued function on $X^{*}$ 
which separates $p$ and $F$. In the other case, if $x_{0} \in q^{-1}(p) \cap X$, let $\varphi_{0}: X \rightarrow \mathbf{R}$ be a continuous real-valued function on $X$ which is zero at $x_{0}$ and one on $q^{-1}(F) \cap X$. For each $y \in M_{f}$ and each $\alpha \in \Lambda_{y}$, choose $\varphi_{\alpha}: p_{y}\left(I_{\alpha}\right) \rightarrow \mathbf{R}$ which is one at

$$
\left(q^{-1}(F) \cap p_{y}\left(I_{\alpha}\right)\right) \cup p_{y}\left(\left[\frac{1}{2}, 1\right]\right) \subset p_{y}\left(I_{\alpha}\right)
$$

and such that $\varphi_{\alpha}\left(p_{y}(1)\right)=\varphi_{0}\left(x_{\alpha}\right)$. These functions combine to form one $\Phi: W \rightarrow \mathbf{R}$ which in turn induces a continuous real-valued function $g$ on $X^{*}$ separating $p$ and $F$.

Adjunction space representation of $X^{*}$. The space $X^{*}$ can also be realized as an adjunction space. For, let $F$ be the closed discrete subset of $\bigoplus Z_{y}$ whose intersection with each $Z_{y}$ is the set $\left\{p_{y}(1) \in p_{y}\left(I_{\alpha}\right): \alpha \in \Lambda_{y}\right\}$. The map $g: F \rightarrow X$ which sends each $p_{y}(1) \in p_{y}\left(I_{\alpha}\right)$ to $x_{\alpha} \in C_{\alpha} \subset f^{-1}(y)$ yields the adjunction space $\oplus Z_{y} \cup_{g} X$ which is easily seen to be homeomorphic to $X^{*}$.

Thus any topological property $P$ which is preserved under disjoint topological sums and adjunctions and is also possessed by each $Z_{y}$ will be enjoyed by $X^{*}$ whenever $X$ enjoys $P$. In particular, $X^{*}$ is normal, hereditarily normal, perfectly normal, (hereditarily) collectionwise normal and (hereditarily) paracompact whenever $X$ is. Moreover, if $X$ is a stratifiable space, or a semi-stratifiable space [6], or a normal $\sigma$-space [29], so is $X^{*}$ as all these properties are enjoyed by metrizable spaces and are preserved under adjunctions ([15] [24]).

Recapitulating, we have the following:

2.2. TheOREM [20]. There is a superspace $X^{*}$ of $X$ containing $X$ as a closed subspace and a monotone function $f^{*}: X^{*} \rightarrow Y$ whose restriction to $X$ is $f$ and $X^{*}$ is (pathwise) connected, or locally (pathwise) connected, or $T_{0}$, $T_{1}, T_{2}, T_{3}$ and $T_{3 \frac{1}{2}}$ whenever $X$ is. Moreover, if $f$ is continuous, so is $f^{*}$. Furthermore, $X^{*}$ is normal, or hereditarily normal, or perfectly normal, or (hereditarily) collectionwise normal, or (hereditarily) paracompact, or a stratifiable space, or a semi-stratifiable space, or a normal $\sigma$-space whenever $X$ is.

2.3. The example of exponential map wrapping the line round the circle shows that $X^{*}$ need not preserve metrizability, either axiom of countability, weight or local weight, separability or density, the Lindelöf property, (countable, sequential, pseudo or local) compactness. Further, this example shows that $X^{*}$ need not preserve the property of being a developable space, or a Moore space, or a $p$-space [1] or $M$-space [28], or 
complete in the sense of Čech [13], countable or pointwise countable type [1], $q$-space [25] or $r$-space [26].

2.4. Let $X$ denote the subset of the plane consisting of

$$
\{(x, y): x+y \leq 1, x, y \geq 0\} \cup(\{1\} \times \mathbf{N}),
$$

where $\mathbf{N}$ denotes the set of natural numbers. Let $Y$ be the real-axis and let $f$ denote the restriction of the natural projection $\pi_{x}$ to $X$. Let $f^{*}: X^{*} \rightarrow Y$ denote the monotone extension discussed in the preceding paragraphs. Then $X^{*}$ is a metrizable space while the extension space of the monotone extension constructed in [20] is not even first countable.

Examples 2.3 and 2.4 suggest the desirability of finding sufficient conditions for preservation of metrizability and other important properties in $X^{*}$. In the following theorem we offer one such sufficient condition.

2.5. TheOREM. If the set $C=\left\{x_{\alpha} \in C_{\alpha} \subset f^{-1}(y): \alpha \in \Lambda_{y}, y \in M_{f}\right\}$ in the construction of $X^{*}$ is a closed and a discrete set, then $X^{*}$ preserves metrizability, local weight, completeness in the sense of Čech, countable or pointwise countable type, and the property of being a q-space or r-space. Further, if neither the cardinality of the set $M_{f}$ nor $\sup \left\{\left|\Lambda_{y}\right|: y \in M_{f}\right\}$ is larger than the weight of $X$, then weight is also preserved in $X^{*}$.

Proof. We first prove that under the given hypothesis the quotient mapping $q: W \rightarrow X^{*}$ is a closed mapping. Suppose that $F$ is a closed subset of $W$ and let $\left\{z_{\alpha}\right\}$ be a net in $q(F)$ converging to a point $z$ in $X^{*}$. Now

$$
q(F)=q(F \cap X) \cup\left(\cup q\left(F \cap Z_{y}\right)\right)
$$

and the restriction of $q$ to $X$ and to each $Z_{y}$ is an embedding. Since $q(X)$ and each $q\left(Z_{y}\right)$ is closed in $X^{*}$, we conclude that $q(F \cap X)$ and each $q\left(F \cap Z_{y}\right)$ is closed in $X^{*}$. Thus if the net $\left\{z_{\alpha}\right\}$ is frequently in $q(F \cap X)$ or in some $q\left(F \cap Z_{y}\right), z$ must belong to $q(F)$ and our proof is complete. If $q^{-1}(z)=\{t\}$ with $t \in Z_{y}$, let $U=Z_{y}$. If $q^{-1}(z)=\{x\}$ with $x \in X$, let $U=X-C$. If $q^{-1}(z)=\left\{x_{\alpha}, 1\right\}$ with $x_{\alpha} \in C_{\alpha} \subset f^{-1}(y)$ and $1 \in p_{y}\left(I_{\alpha}\right)$ $\subset Z_{y}$, let $U$ be the union of $Z_{y}$ and a neighbourhood $V$ of $x_{\alpha}$ in $X$ such that $V$ contains no other element of the set $C$. In any case $q(U)$ is a neighbourhood of $z$ which has a nonempty intersection with at most one $q\left(F \cap Z_{y}\right)$. But $\left\{z_{\alpha}\right\}$ is eventually in $q(U)$, and hence is frequently in either

$$
q(U) \cap q(F \cap X) \text { or } q(U) \cap q\left(F \cap Z_{y}\right) .
$$

Hence $q$ is a closed mapping. Since each $q^{-1}(z)$ is at most a doubleton, $q$ is a finite-to-one closed mapping. Thus, any topological property which is 
preserved under finite-to-one closed mappings, disjoint topological sums and possessed by $X$ and each $Z_{y}$ will be enjoyed by $X^{*}$. Since all the properties listed in the statement of Theorem 2.5 except local weight are preserved under finite-to-one closed mappings, disjoining topological sums and are possessed by each $Z_{y}$, they are preserved in $X^{*}$.

To see preservation of local weight in $X^{*}$, let $m$ denote the larger of the weight of $X$ and $\boldsymbol{\aleph}_{0}$ and let $z \in X^{*}$. If $q^{-1}(z)=\{t\}$ where $t \in Z_{y}$, then the image of a base at $t$ under $q$ is a base at $z$. If $q^{-1}(p)=\{x\}$ where $x \in X$, a base at $x$ may be chosen such that each member of which is contained in $X-C$. The image of this base under $q$ is a base at $z$. If $q^{-1}(z)=\left\{x_{\alpha}, 1\right\}$ with $x_{\alpha} \in C_{\alpha} \subset f^{-1}(y)$ and $1 \in p_{y}\left(I_{\alpha}\right) \subset Z_{y}$, choose a base $\beta$ at $x$ whose members contain at most one member of $C$, and choose a base $\mathcal{V}$ at $1 \in p_{y}\left(I_{\alpha}\right) \subset Z_{y}$, with cardinality of $\mathscr{B}$ and $\mathcal{V}$ no larger than $m$. The images under $q$ of the sets of the form $B \cup V$ with $B \in \mathscr{B}$ and $V \in \mathcal{V}$, form a base at $z$ of cardinality no larger than $m$.

For what remains we need only note that

$$
\text { Weight } \begin{aligned}
W & =\text { weight } X+\left|M_{f}\right| \cdot \sup \left\{\left|\Lambda_{y}\right|: y \in M_{f}\right\} \cdot \boldsymbol{\aleph}_{0} \\
& \leq m+m \cdot m \cdot \boldsymbol{\aleph}_{0}=m
\end{aligned}
$$

and that perfect mappings do not increase weight.

2.6. REMARK. Example 2.3 shows that the restrictions imposed on the choice set $C$ in Theorem 2.5 are not superfluous. Simple examples suffice to show that they cannot be weakened even to $C$ being a countable discrete set. For let $X$ denote the subset of the plane consisting of

$$
\{(x, y): x, y \geq 0, x+y \leq 1\} \cup\left\{\left(1, \frac{1}{n}\right): n \in \mathbf{N}\right\}
$$

and let $f$ be the restriction of the natural projection $\pi_{x}$ to $X$. Then the monotone extension space $X^{*}$ fails to be first countable.

At this stage it seems natural to ask: Are the restrictions imposed on the choice set $C$ in Theorem 2.5 necessary? This question bears an affirmative answer for metrizability, either axiom of countability and local compactness. For, if $X$ is first countable and the choice set has a limit point, then it is easily shown that the space $X^{*}$ contains a subspace which is homeomorphic to the non-first countable (infact, non-Fréchet) space $M$ of [11, Example 5.1] and thus fails to possess any property which implies first countability. A similar argument suffices to show the nonlocal compactness of the space $X^{*}$ in case the choice set $C$ possesses a limit point. 
3. Mappings with fibres having countably many components. The space $X^{*}$ in Example 2.4 is not locally compact. We do not know of a monotone extension which preserves the property of being a locally compact Hausdorff space. The monotone extension discussed in [22] preserves compactness as well as local compactness but its extension space fails to be Hausdorff. However, if each fibre has at most countably many components we suggest a modification of the technique of the preceding section which may preserve local compactness in certain specific situations.

\section{CONSTRUCTION B}

Let $y \in M_{f}$ and let $f^{-1}(y)=\cup C_{i}$, where each $C_{i}$ is a component of $f^{-1}(y)$. Define $\Sigma_{y}=\mathbf{N}$ if $f^{-1}(y)$ is the union of infinitely many components and $\Sigma_{y}=\{1,2, \ldots, n\}$ if $f^{-1}(y)$ consists of $n(>1)$ components. Let $\Sigma_{y}^{0}$ be $\mathbf{N}$ or $\{1, \ldots, n-1\}$ according as $\Sigma_{y}$ is $\mathbf{N}$ or $\{1, \ldots, n\}$. Let $\Sigma$ denote the disjoint union of the sets $\Sigma_{y}^{0}, y \in M_{f}$. For each $y \in M_{f}$ and for each $i \in \Sigma_{y}$, choose $x_{i} \in C_{i} \subset f^{-1}(y)$. For each $j \in \Sigma$, let $I_{j}$ denote a copy of $I$. Let $W_{1}=X \oplus\left(\oplus I_{j}\right)$, where the second disjoint topological sum is taken over the set $\Sigma$. By identifying the end points 0 and 1 of each $I_{j}$ (considered as a subset of $\left.W_{1}\right)\left(j \in \Sigma_{y}^{0}\right)$ with $x_{j}$ and $x_{j+1}$ (as points of $f^{-1}(y) \subset X \subset$ $W_{1}$ ), respectively, we arrive at a quotient space $\hat{X}$ of $W_{1}$. The inclusion mapping $i: X \rightarrow W_{1}$ composes with the quotient mapping $p: W_{1} \rightarrow \hat{X}$ to give a closed embedding of $X$ into $\hat{X}$. Thus we may consider $\hat{X}$ as a superspace of $X$ containing $X$ as a closed subspace.

Let $f_{2}$ be the mapping of $W_{1}$ into $Y$ whose restriction to $X$ is $f$ and whose restriction to each $I_{j}$ with $j \in \Sigma_{y}^{0}$ is the constant mapping onto $\{y\}$. We leave it to the reader to verify that the unique function $\hat{f}: \hat{X} \rightarrow Y$ satisfying $f_{2}=\hat{f} \circ p$ is a monotone extension of $f$ and that all the properties listed in Theorem 2.2 are carried over from $X$ to $\hat{X}$.

We may point out that the space $\hat{X}$ can also be realized as an adjunction space. For, let $F$ be the closed discrete subset of $\oplus I_{j}$ whose restriction with each $I_{j}$ is the set $\{0,1\}$. The map $h: F \rightarrow X$ which sends each $0 \in I_{j}\left(j \in \Sigma_{y}^{0}\right)$ to $x_{j} \in C_{j} \subset f^{-1}(y)$ and $1 \in I_{j}\left(j \in \Sigma_{y}^{0}\right)$ to $x_{j+1} \in$ $C_{j+1} \subset f_{\hat{X}}^{-1}(y)$ yields the adjunction space $\bigoplus I_{j} \cup_{h} X$ which is homeomorphic to $\hat{X}$.

Now, let $f: X \rightarrow Y$ be the same as in Example 2.4 and let $\hat{f}: \hat{X} \rightarrow Y$ be the monotone extension defined as above in $\S 3$, Construction $\mathrm{B}$. Then the space $\hat{X}$ is a locally compact separable metrizable space while $X^{*}$ fails to be locally compact. 
Further, we leave it to the reader to verify that the analogue of Theorem 2.5 remains true for $\hat{X}$ if the choice set in the construction of $\hat{X}$ is closed and discrete.

4. Monotone extensions with compact fibres. In this section, we shall modify the technique of preceding sections so as to obtain monotone extensions with additional property that the point inverses are compact.

Construction. In the construction of the space $X^{*}$ in $\$ 2$ replace the step II by Step II': Let $A_{f}$ denote the set $M_{f} \cup C_{f}$. For each $y \in M_{f}$, let $A_{y}$ denote $\beta Z_{y}$, the Stone-Cech compactification of the space $Z_{y}$. For each $y \in A_{f}-M_{f}$, let $A_{y}$ denote a compactification of the fibre $f^{-1}(y)$ (e.g., if $X$ is a Tychonoff space, $A_{y}$ may be taken to be the Stone-Čech compactification of $\left.f^{-1}(y)\right)$.

Let $W_{2}=X \oplus\left(\oplus A_{y}\right)$, where the second disjoint topological sum is taken over the set $A_{f}$. First for each $y \in M_{f}$, identify each $p_{y}(1) \in p_{y}\left(I_{\alpha}\right)$ ( $\alpha \in \Lambda_{y}$ ) (thought of as a point of $Z_{y} \subset A_{y} \subset W_{2}$ ) with $x_{\alpha} \in C_{\alpha} \subset f^{-1}(y)$ (as a point of $X \subset W_{2}$ ). Subsequently, for each $y \in A_{f}-M_{f}$, identify each $x \in f^{-1}(y)$ (thought of as a point of $X \subset W_{2}$ ) with the corresponding $x \in f^{-1}(y) \subset A_{y} \subset W_{2}$. Let $\tilde{X}$ be the resultant quotient space. The inclusion mapping $i: X \rightarrow W_{2}$ composes with the quotient mapping $r: W_{2} \rightarrow \tilde{X}$ to give an embedding of $X$ into $\tilde{X}$. Thus $\tilde{X}$ may be considered as a superspace of $X$.

Let $f_{2}$ be the mapping of $W_{2}$ into $Y$ whose restriction to $X$ is $f$ and whose restriction to each $A_{y}$ is the constant mapping onto $\{y\}$. Then the unique mapping $\tilde{f}: \tilde{X} \rightarrow Y$ satisfying $f_{2}=\tilde{f} \circ r$ is a monotone extension of $f$ such that each point inverse is a continuum.

5. Applications. The device of monotone extensions of mappings developed in the preceding sections is a useful tool in weakening the hypothesis of monotoneity (to a certain extent) in various results in the lore of literature on monotone mappings. In this section, we apply the technique of monotone extensions to extend certain results of Whyburn ([32], [33] [34]) pertaining to monotone and compact mappings. In fact, we show that in certain results of Whyburn the hypothesis of monotoneity may be replaced by a weaker hypothesis: monotone except at finitely many points and such that each point inverse has at most finitely many components.

Throughout this section, all the mappings are assumed to be continuous and all the spaces are assumed to be Hausdorff. 
Let $f: X \rightarrow Y$ be a mapping. Then a set $A$ contained in $X$ is called a trace of a set $B$ in $Y$ if $f(A)=B$. The mapping $f$ is said to have compact trace property if each compact set in $Y$ is the image of a compact set in $X$ under $f$.

Let $Q^{\prime}$ be the union of the interiors of images of all compact sets in $X$ ( = the set of all points which lie in the interior of image of a compact set). Let $Q$ denote the union of the interior of all compact sets in $Y$ having compact inverse. Let $P^{\prime}=f^{-1}\left(Q^{\prime}\right)$ and $P=f^{-1}(Q)$. It is immediate that the sets $P, P^{\prime}, Q$ and $Q^{\prime}$ are open, the mapping $f \mid P$ is compact and the mapping $f \mid P^{\prime}$ has compact trace property.

Whyburn [32] showed that for any surjective mapping between locally compact separable metric spaces the set $Q^{\prime}$ is nonempty. Essentially the same proof yields the following extension of Whyburn's result.

5.1. Proposition. Let $f: X \rightarrow Y$ be a mapping of a $\sigma$-compact space $X$ onto a Bair space $Y$. Then $Q^{\prime}$ is nonempty.

From here onward all the mappings are assumed to satisfy the condition that point inverses are compact, unless explicitly stated otherwise.

5.2. TheOREM. Let $f: X \rightarrow Y$ be a quotient mapping from a locally compact space $X$ onto a space $Y$ such that $f$ is monotone except at finitely many points and each point inverse has at most finitely many components. Then $f$ is a closed mapping and $Y$ is also locally compact.

5.3. THEOREM. Let $f: X \rightarrow Y$ be a mapping from a locally compact space $X$ onto a space $Y$ such that $f$ is monotone except at finitely many points and each point inverse has at most finitely many components. Then any compact set $H$ in $Y$ which has a compact trace $K$ has compact inverse.

5.4. THEOREM. Let $f: X \rightarrow Y$ be a mapping from a locally compact space $X$ onto $Y$ such that $f$ is monotone except at finitely many points and each point inverse has at most finitely many components. Then $f$ is compact if and only if $f$ has compact trace property.

We recall that a generalized continuum is a connected separable metric space which is locally compact. 
5.5. THEOREM. Let $X$ and $Y$ be locally connected generalized continua having the property that there is an integer $k \geq 1$ such that the complement of each compact set in $X$ or $Y$ has exactly $k$ non-conditionally compact components. Let $f: X \rightarrow Y$ be a mapping such that $f$ is monotone except at finitely many points and each point inverse has at most finitely many components. If each $y \in Y$ has a base of neighbourhoods whose boundaries have compact traces, then $f$ is a perfect mapping.

Theorems 5.2-5.5 are extensions of Whyburn's results ([32] [33] [34]) who first proved them under the hypothesis that $f$ is a monotone mapping. Now we sketch the proofs of Theorems 5.2-5.5.

If $f: X \rightarrow Y$ is a mapping on a locally compact space $X$ such that the set $M_{f}$ of non-monotone points of $f$ is finite (i.e., $f$ is monotone except at finitely many points) and each point inverse has at most finitely many components, then $f^{*}: X^{*} \rightarrow Y$ is a monotone mapping having compact point inverses and $X^{*}$ is a locally compact space (the needed Hausdorff axiom also lifts from $X$ to $X^{*}$ ). Now since $f$ in 5.2 is a quotient mapping, so is $f^{*}$. The conclusion of 5.2 follows in view of Whyburn's original result [34, Theorem 3], the fact that $X$ is a closed subspace of $X^{*}$ and that the restriction of a closed mapping to a closed subspace is closed.

The conclusions of Theorems 5.3 and 5.4 follow in view of Whyburn's original results [32, Theorems $2.6,2.61]$ and the fact that a closed set meets a compact set in a compact set. Further, for Theorem 5.5 we need only note that if $f, X$ and $Y$ satisfy the hypothesis of Theorem 5.5, then $f^{*}$, $X^{*}$ and $Y$ satisfy the hypothesis of Whyburn's result [32, Theorem 3.3] and that the restriction of a perfect mapping to a closed subspace is a perfect mapping.

Simple examples can be given to show that the hypothesis of monotoneity in Theorems 5.2-5.4 is quite essential though point inverses are compact. For, if $X$ is the part of parabola $y^{2}=x$ for $-1<y \leq 1$, the vertical projection of $X$ onto the closed unit interval $[0,1]$ satisfies all the conditions of Theorems 5.2-5.4 except monotoneity and yet neither $f$ is closed nor $[0,1]$ has compact inverse though $[0,1]$ has compact trace.

5.6. THEOREM. Let $X$ be a locally compact space such that every open subspace of $X$ is $\sigma$-compact. If $f: X \rightarrow Y$ is a mapping from $X$ onto a Bair space $Y$, then $Q^{\prime}$ is dense in $Y$ (point inverses are not necessarily required to be compact for this). Moreover, if $f$ is monotone except at finitely many points and each point inverse has at most finitely many components, then $Q=Q^{\prime}$. Further, if $f$ is one-to-one, then $f$ maps $P$ topologically onto $Q$.

Proof. Let $V$ be a nonempty open subset of $Y$. Then $f^{-1}(V)$ is open and thus by hypothesis on $X, f^{-1}(V)=\cup_{n=1}^{\infty} K_{n}$, where each $K_{n}$ is 
compact. Since the property of being a Bair space is open hereditary and since $V=\cup_{n=1}^{\infty} f\left(K_{n}\right)$, for some integer $m$, int $V f\left(K_{m}\right) \neq \varnothing$. By openness of $V$, int $_{V} f\left(K_{m}\right)=\operatorname{int}_{Y} f\left(K_{m}\right)$ and hence $Q^{\prime}$ is dense in $Y$. If $f$ is monotone except at finitely many points and each point inverse has at most finitely many components, by Theorem 5.3, it follows that $Q=Q^{\prime}$.

To prove last assertion, let $y \in Q$. There is a compact set $K$ in $Y$ such that $y \in$ int $f(K)$ and $f^{-1}(K)$ is compact. Then $f \mid f^{-1}(K)$ is a one-to-one closed mapping onto $K$ and hence a homeomorphism. Thus $f \mid f^{-1}$ (int $K$ ) is a homeomorphism and consequently $f \mid P$ maps $P$ topologically onto $Q$.

Whyburn first obtained Theorem 5.6 for monotone mappings between locally compact separable metric spaces (see [33, p. 172]) and later for monotone mappings with domain as locally compact second countable space and range as a complete metric space (see [34, Theorem B]). Thus, in particular, it follows that in the original results of Whyburn instead of monotoneity it is sufficient to require that $f$ is monotone except at finitely many points with point inverses having at most finitely many components.

We may point out that in the first halves of each of Theorems 5.6 and the original Theorems of Whyburn, mentioned in the above paragraph, the hypothesis that point inverses are compact is not required. It is essential only in the later halves in conjunction with monotoneity.

Finally, the author wishes to thank the referee for helpful suggestions.

\section{REFERENCES}

1. A. V. Arhangel'skii, $A$ class of space containing all metric and all locally bicompact spaces, Soviet Math., 1 (1963), 1051-1055.

2. H. Bauer, Konservative Abbildungen local kompakter Raüme, Math. Ann., 138 (1959), 398-427.

3. G. L. Cain Jr., Compactifications of mappings, Proc. Amer. Math. Soc., 23 (1969), 298-303.

4. __ Extensions of compactifications of mapping, Math. Ann., 191 (1971), 333-336.

5. __ Metrizable mapping compactifications, General Topology and Appl., 2 (1972), 271-275.

6. G. Creede, Semi-stratifiable spaces, Topology Conference Arizona University, (1967), 318-324.

7. F. A. Delhan and G. E. Strecker, A simplified approach to compactifications of mappings, Bull. Amer. Math. Soc., 79 (1973), 1030-1032.

8. R. F. Dickman Jr., Unified spaces and singular sets of mappings of locally compact spaces, Fund. Math., 62 (1968), 103-123.

9. $326-332$.

, On closed extensions of functions, Proc. Nat. Acad. Sci. U.S.A., 62 (1969),

10. Regular closed maps, Proc. Amer. Math. Soc., 39 (1973), 414-416.

11. S. P. Franklin, Spaces in which sequences suffice. II, Fund. Math., 61 (1967), 51-56. 
12. S. P. Franklin and J. K. Kohli, On open extensions of maps, Canad. J. Math., 22 (1970), 691-696.

13. Z. Frolik, Generalization of $G_{\delta}$ property of metric spaces, Czechoslovak J. Math., 10 (1960), 359-379.

14. H. Herrlich, Topologische Reflexionen Und Co-reflexionen, (Springer Verlag, Berlin, 1968).

15. S. T. Hu, Theory of Retracts, Wayne State Univ. Press, Detroit Mich., 1965.

16. J. K. Kohli, Open extensions of functions, Ph.D. Thesis, Indian Institute of Technology, Kanpur (1970).

17. __ A note on open extensions of maps, Canad. J. Math., 24 (1972), 1139-1144.

18. Finite-to-one maps and open extensions of maps, Proc. Amer. Math. Soc., 48 (1975), 464-468.

19. L_ Local topological properties of maps and open extensions of maps, Canad. J. Math., 29 (1977), 1121-1128.

20. On Hausdorff monotone extensions of maps, J. London Math. Soc., 15 (1977), $547-550$.

21. A class of spaces containing all connected and all locally connected spaces, Math. Nachr., 82 (1978), 121-129.

22. __ A unified approach to monotone extensions of maps, Acta Math. Acad. Sci. Hungar., 36 (1-2) (1980), 189-194.

23. N. Krolevec, Locally perfect mappings, Soviet Math. Dokl., 8 (1967), 964-967.

24. B. H. McCandles, Adjunction spaces and hereditary property, Proc. Amer. Math. Soc., 18 (1967), 695-700.

25. E. A. Michael, $A$ note on closed maps and compact sets, Israel J. Math., 2 (1964), $173-176$.

26. __ $\boldsymbol{\aleph}_{0}$-spaces, J. Math. Mech., 15 (1966), 983-1002.

27. R. C. Moore and S. G. Mrówka, Topologies determined by countable objects, Notices Amer. Math. Soc., 11 (1964), 554.

28. K. Morita, Products of normal spaces with metric spaces, Math. Ann., 154 (1964), $365-382$.

29. A. Okuyama, Some generalization of metric spaces, their metrization theorems and product spaces, Sci. Rep. Tokyo Kyoku Daigaku, Sec. A., 9 (1967), 236-254.

30. I. A. Vainstein, On closed mappings, Moskov. Gos. Univ. Zap. 155 Mat., 5 (1952), $3-53$.

31. .G. T. Whyburn, $A$ unified space for mappings, Trans. Amer. Math. Soc., 74 (1953), $344-350$.

32.

33.

34. , Compactness of certain mappings, Amer. J. Math., 81 (1959), 306-314. , Compactifications of mappings, Math. Ann., 166 (1966), 168-174. , Dynamic topology, Amer. Math. Monthly, 77 (1970), 556-570.

Received May 22, 1981 and in revised form September 30, 1981. This research was partially supported by a grant from University Grants Commission, New Delhi, India.

Hindu College

UNIVERSITY OF DELHI

DELHI-110007

INDIA

Current address: Lamar University

Beaumont, TX 77710 



\section{PACIFIC JOURNAL OF MATHEMATICS}

EDITORS

Donald BABBITT (Managing Editor)

University of California

Los Angeles, CA 90024

Hugo Rossi

University of Utah

Salt Lake City, UT 84112

C. C. Moore and Arthur Ogus

University of California

Berkeley, CA 94720
J. DugunduI

Department of Mathematics

University of Southern California

Los Angeles, CA 90089-1113

R. FinN and H. SAmelson

Stanford University

Stanford, CA 94305

\section{ASSOCIATE EDITORS}
R. ARENS
E. F. BECKENBACH
B. H. NEUMANN
F. WolF
K. YoshidA (1906-1982)

\section{SUPPORTING INSTITUTIONS}

UNIVERSITY OF ARIZONA

UNIVERSITY OF BRITISH COLUMBIA

CALIFORNIA INSTITUTE OF TECHNOLOGY

UNIVERSITY OF CALIFORNIA

MONTANA STATE UNIVERSITY

UNIVERSITY OF NEVADA, RENO

NEW MEXICO STATE UNIVERSITY

OREGON STATE UNIVERSITY
UNIVERSITY OF OREGON

UNIVERSITY OF SOUTHERN CALIFORNIA

STANFORD UNIVERSITY

UNIVERSITY OF HAWAII

UNIVERSITY OF TOKYO

UNIVERSITY OF UTAH

WASHINGTON STATE UNIVERSITY

UNIVERSITY OF WASHINGTON 


\section{Pacific Journal of Mathematics}

Vol. 105, No. 1 September, 1983

Kenneth F. Andersen, On the transformation of Fourier coefficients of

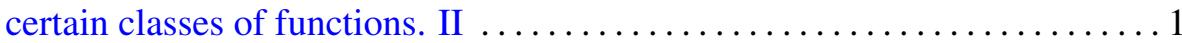

Gavin Brown, Irving Leonard Glicksberg and Edwin Hewitt, Indicator functions with large Fourier transforms $\ldots \ldots \ldots \ldots \ldots \ldots \ldots \ldots \ldots \ldots$

Shih-Sen Chang, Some random fixed point theorems for continuous random

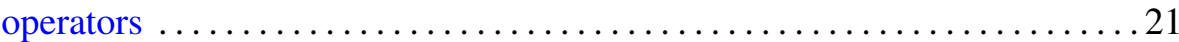

K. C. Chattopadhyay and Olav Njstad, Quasiregular nearness spaces and

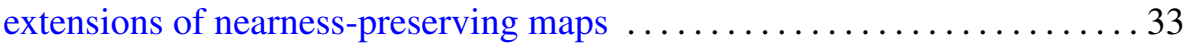

Thomas W. Cusick, The two-dimensional Diophantine approximation

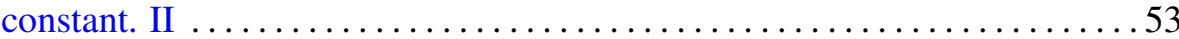

Eric Karel van Douwen and Jan van Mill, Spaces without remote points . . .669 Hector O. Fattorini, Convergence and approximation theorems for

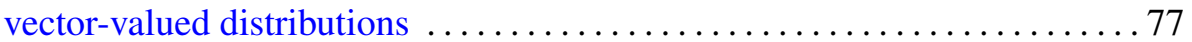

John J. F. Fournier and Louis Pigno, Analytic and arithmetic properties of

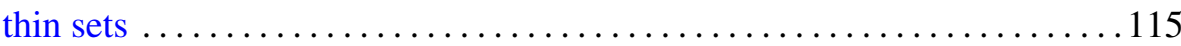

David Goss, On a new type of $L$-function for algebraic curves over finite

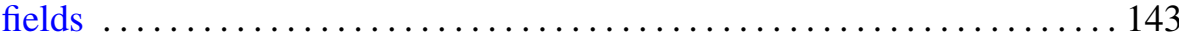

Douglas Austin Hensley, Lattice vertex polytopes with interior lattice

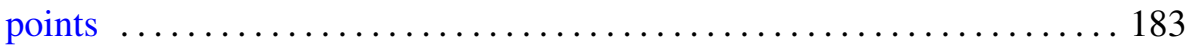

J. K. Kohli, Monotone extensions of mappings and their applications ...... 193

John C. Morgan, II, On equivalent category bases . . . . . . . . . . . . 207

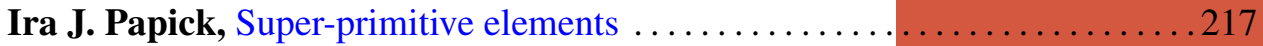

José Luis Rubio de Francia and José Luis Torrea, Vector extensions of

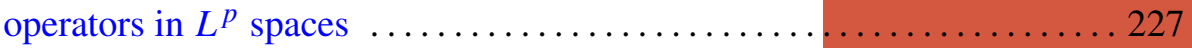

Mark Phillip Thomas, Closed ideals of $l^{1}\left(\omega_{n}\right)$ when $\left\{\omega_{n}\right\}$ is star-shaped $\ldots 237$ 